\title{
The origin of metallicity gradients in massive galaxies at large radii
}

\author{
Michaela Hirschmann ${ }^{1}$ and Thorsten $\mathrm{Naab}^{2}$ \\ ${ }^{1}$ UPMC-CNRS, UMR7095, Institut dAstrophysique de Paris, F-75014 Paris, France \\ email: hirschma@iap.fr \\ ${ }^{2}$ Max-Plank-Institute für Astrophysik, Karl-Schwarzschild Strasse 1, D-85740 Garching, \\ Germany \\ email: naab@mpa-garching.mpg.de
}

\begin{abstract}
We investigate the origin of stellar metallicity gradients in massive galaxies at large radii $\left(r>2 R_{\text {eff }}\right)$ using ten cosmological zoom simulations of halos with $6 \times 10^{12} M_{\odot}<M_{\text {halo }}<$ $2 \times 10^{13} M_{\odot}$. The simulations follow metal cooling and enrichment from SNII, SNIa and AGB winds. We explore the differential impact of an empirical model for galactic winds that reproduces the evolution of the mass-metallicity relation. At larger radii, the galaxies become more dominated by stars accreted from satellite galaxies in major and minor mergers. In the wind model, fewer stars are accreted, but they are significantly more metal poor resulting in steep global metallicity $\left(\left\langle\nabla Z_{\text {stars }}\right\rangle=-0.35 \mathrm{dex} / \mathrm{dex}\right)$ gradients in agreement with observations. Metallicity gradients of models without winds are inconsistent with observations. For the wind model, stellar accretion is steepening existing in-situ metallicity gradients by about 0.2 dex by the present day and is required to match observed gradients. Metallicity gradients are significantly steeper for systems, which have accreted stars in minor mergers. In contrast, galaxies with major mergers have relatively flat gradients, confirming previous results. We highlight the importance of stellar accretion for stellar population properties of massive galaxies at large radii, which provide important constraints for formation models.
\end{abstract}

Keywords. galaxies: evolution - galaxies: formation - methods: numerical

\section{Introduction}

It is a natural prediction of modern hierarchical cosmological models that the assembly of massive galaxies involves major and minor mergers although most stars in most galaxies have been made in-situ from accreted or recycled gas. Nonetheless, these mergers are expected to play a significant role for the structural and morphological evolution of the massive early-type galaxy population. One important structural galaxy property, which is thought to be strongly influenced by mergers, are the (in general negative) metallicity gradients observed early-on in massive, present-day elliptical (e.g. McClure \& Racine 1969), but also in disk galaxies (e.g. Wyse \& Silk 1989), typically within $1 R_{\text {eff }}$. Thanks to improved and more elaborated observational techniques, present-day metallicity gradients can nowadays be measured out to much larger radii, partly out to even $8 R_{\text {eff }}$, (e.g. La Barbera et al. 2012).

Previous studies (e.g. Kobayashi 2004) only discuss the emergence of inner gradients (up to $3 R_{\text {eff }}$ ) at comparably poor spatial resolution. Here, we focus on the stellar accretion origin of metallicity gradients in high-resolution re-simulated massive galaxies at large radii $\left(2 R_{\text {eff }}<r<6 R_{\text {eff }}\right)$ in a full cosmological context. We particularly intend to explore the combined effect of strong galactic winds and of the individual merger and accretion histories on the in-situ formed and accreted stellar fractions and on the steepening/flattening of the metallicity gradients at these large radii. 

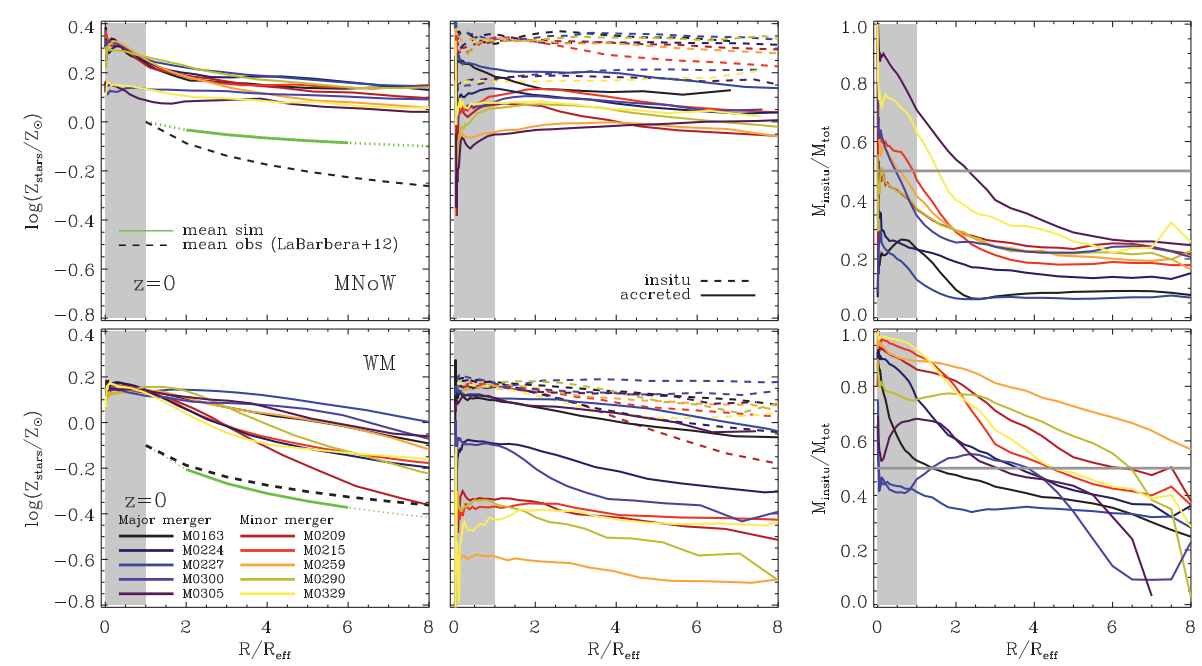

Figure 1. Left column: Total stellar metallicity gradients (mass weighted) at $z=0$ for the ten main galaxies (different colors) simulated with the $\mathrm{MNoW}$ and $\mathrm{WM}$ model. The green solid lines indicate the average gradient at $2<R / R_{\mathrm{eff}}<6$. Middle panels: Metallicity gradients at $z=0$ separated into stars formed in-situ (dashed lines) and accreted stars (solid lines). Right panels: Fraction of in-situ to total stellar mass as a function of radius.

\section{High-resolution simulations of individual galaxy halos}

We consider the 10 most massive high-resolution, cosmological zoom simulations (covering a mass range of $6 \times 10^{12}<M_{\text {halo }}<2 \times 10^{13} M_{\odot}$ ) presented in (Hirschmann et al. 2013) including a treatment for metal enrichment (SNII, SNIa and AGB stars) and a phenomenological feedback scheme for galactic winds (Oppenheimer \& Dave 2006, 2008). The dark matter/gas particles have a mass resolution of $m_{\mathrm{dm}}=2.5 \cdot 10^{7} M_{\odot} h^{-1}$ and $m_{\text {gas }}=m_{\text {star }}=4.2 \cdot 10^{6} M_{\odot} h^{-1}$, respectively with a co-moving gravitational softening length for the gas and star particles of $400 h^{-1} \mathrm{pc}$ and for the dark matter particles of $890 \mathrm{~h}^{-1} \mathrm{pc}$ (Oser et al. 2010).

These cosmological zoom simulations were shown to be successful in suppressing early star formation at $z>1$, in predicting reasonable star formation histories for galaxies in present day halos of $\sim 10^{12} M_{\odot}$, in producing galaxies with high cold gas fractions (30 - 60 per cent) at high redshift, and in significantly reducing the baryon conversion efficiencies for halos $\left(M_{\text {halo }}<10^{12} M_{\odot}\right)$ at all redshifts in overall good agreement with observational constraints. Due to the delayed onset of star formation in the wind models, the metal enrichment of gas and stars is delayed and is also found to agree well with observational constraints.

\section{Metallicity gradients of present-day massive galaxies}

In Fig. 1, we show the total (mass-weighted) stellar metallicity gradients out to $8 R_{\text {eff }}$ for the main galaxies at $z=0$ in the MNoW and the WM model (as indicated in the legend). For the MNoW galaxies, the (most) central metallicity has values of $Z / Z_{\odot} \sim 0.4$ and drops to $Z / Z_{\odot} \sim 0.1$ at large radii, the slopes reach a minimum value of -0.25 $\mathrm{dex} / \mathrm{dex}$. This gradient is mainly driven by the accreted stars (the in-situ distributions are almost all nearly flat), which have on average solar metallicity (top middle panel of Fig. 1) and dominate most systems outside $1 R_{\text {eff }}$ (top right panel of Fig. 1). 
The WM galaxies (bottom panels of Fig. 1) have lower central metallicities $\left(Z / Z_{\odot} \sim\right.$ 0.2 ) with much steeper outer gradients down to $-0.76 \mathrm{dex} / \mathrm{dex}$ with a mean of $-0.35 \mathrm{dex} /$ dex. The reason for the steeper gradients in the WM compared to the MNoW model is twofold: on the one hand, the steeper gradients originate from the accretion of metalpoorer stellar populations. On the other hand, also the in-situ components show metallicity gradients contributing to the overall gradients. The latter is most likely due to infall of (particularly re-infall of previously ejected) metal-poor gas onto the galaxy which can be then turned into metal-poor stars as a consequence of an inside-out growth, the same process causing the metallicity gradients in disk galaxies. Late re-accretion of previously ejected gas occurs typically in the WM model due to the strong galactic winds, but not in the MNoW model, where the in-situ gradients are, therefore, relatively flat (see top middle panel of Fig. 1). However, despite of the partly negative in-situ gradients, we find that on average, the in-situ gradients are reduced by $\sim 0.2$ dex due to accretion of metal-poor stellar populations.

\subsection{Galaxy mergers and gradients}

The black-blue lines in Fig. 1 indicate galaxies which experienced at least one major galaxy merger since $z=1$, while the red-yellow lines illustrate those having undergone only minor galaxy mergers. Present-day galaxies having experienced a recent major

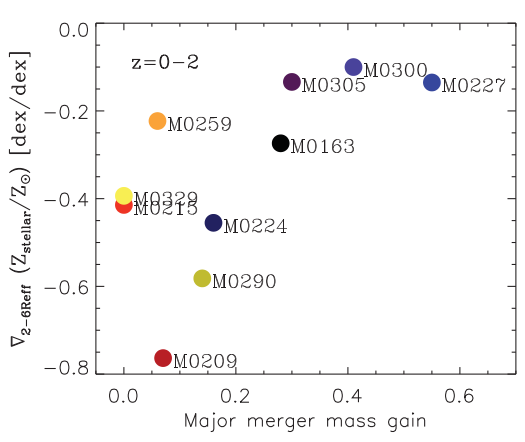

Figure 2. Stellar metallicity gradients (between $2-6 R_{\text {eff }}$ ) versus the mass gain through major mergers for the ten re-simulated galaxies in the WM simulations. The higher the major merger mass gain, the smaller is the slope of the metallicity gradient. merger have typically flatter gradients than those with a more quiet merger history.

Fig. 2 quantifies the connection between the galaxy merger history and the steepness of the metallicity gradients: we show the fitted metallicity gradients at $z=0$ for WM galaxies versus the mass gain by major mergers. The mass gain by major mergers considers the entire stellar mass which was brought into the main galaxy by major mergers since $z=2$ normalised to the present-day stellar mass.

The fitted metallicity gradients correlate with the past merger history: for a major merger mass gain above $20 \%$, the metallicity gradients are flatter than $-0.3 \mathrm{dex} / \mathrm{dex}$. Instead, for lower $\mathrm{x}$-values, the metallicity gradients are mostly more negative than $-0.4 \mathrm{dex} / \mathrm{dex}$. This is due to the fact that the accreted stars show a huge variety of metallicities from $Z / Z_{\odot} \sim-0.6$ to $Z / Z_{\odot} \sim+0.1$ (see bottom middle panel of Fig. 1) depending on the exact merger history: in case of a recent major merger, the accreted metallicity is significantly larger than without a major merger (as more massive galaxies have higher metallicity). The higher metallicity of the accreted stars together with the different mixing behaviour (violent relaxation) in case of major mergers flattens the total metallicity gradients.

This result bears an important implication for observations, as it can help to reconstruct the past assembly history for observed present-day metallicity gradients: The relation between the steepness of the gradients and the individual merger history implies that observed massive galaxies having steep outer gradients most likely have not experienced any major merger event after $z=1$, but instead several minor mergers. 


\subsection{Comparison with observations}

In a recent study of Pastorello et al. (2014), using the SLUGGS survey, they investigate metallicity gradients at least out to $2.5 R_{\text {eff }}$. For comparable stellar masses, their galaxies reveal slopes between $-1.15-+0.18 \mathrm{dex} / \mathrm{dex}$. In addition, in a study by La Barbera et al. (2012), they investigate metallicity gradients of early-type galaxies $\left(M_{\text {stellar }}>\right.$ $\left.3 \times 10^{10} M_{\odot}\right)$ even out to $8 \times R_{\text {eff }}$ using the SDSS-based Spider survey. For massive galaxies with $10^{11}<M_{\text {stellar }}<7 \times 10^{11} M_{\odot}$ they find outer metallicity gradients $\left(1-8 \times R_{\text {eff }}\right)$ in the range of $-0.29--0.74 \mathrm{dex} / \mathrm{dex}$ depending on the stellar population model (illustrated by the black dashed lines in Fig 1).

Overall, our WM galaxies are able to cover such a broad range of slopes $(-0.8-$ $-0.1 \mathrm{dex} / \mathrm{dex}$ ) much better than the MNoW galaxies whose slopes are on average too flat (within the range of $-0.25-+0.03 \mathrm{dex} / \mathrm{dex}$ ). The average metallicity gradient of the WM galaxies $(-0.35 \mathrm{dex} / \mathrm{dex})$ is in excellent agreement with the one of La Barbera et al. (2012). As expected a priori, this implies that a strong stellar feedback is a key mechanism to be consistent with observed steep metallicity gradients in massive galaxies in the local Universe.

\section{Conclusions}

Analysing zoom simulations of 10 massive galaxies suggests that the outer negative metallicity gradients (at radii $r>2 R_{\text {eff }}$ ) of present-day massive galaxies are mainly determined by the accretion of stars with lower metallicity. Towards low redshift, stars accreted in low metallicity galaxies become more and more dominant at large radii and the metallicity gradients of in-situ formed stars in the wind model are enhanced by $\sim 0.2 \mathrm{dex} / \mathrm{dex}$ by accretion of metal-poor systems. The model with galactic winds predicts steeper total metallicity gradients (on average $-0.35 \mathrm{dex} / \mathrm{dex}$ ) as the accreted stellar systems are significantly more metal-poor and despite of the fact that much less stellar mass in total is accreted compared to the no-wind model. We can conclude that stellar accretion (in minor mergers) of low mass satellites results in steep outer metallicity gradients as predicted from idealized experiments (Villumsen 1983) successfully matching the broad range of observed metallicity profiles of local galaxies at large radii.

\section{Acknowledgements}

MH acknowledges financial support from the European Research Council via an Advanced Grant under grant agreement no. 321323NEOGAL. TN acknowledges support from the DFG excellence cluster "Origin of the Universe".

\section{References}

Hirschmann, M., et al. 2013, MNRAS, 436, 2929

Kobayashi, C., 2004, MNRAS, 347, 740

La Barbera, F., et al. 2012, MNRAS, 426, 2300

McClure, R. \& Racine R. 1969, AJ, 74, 1000

Oppenheimer, B. \& Dave, R. 2006, MNRAS, 373, 1265

Oppenheimer, B. \& Dave, R. 2008, MNRAS, 387, 577

Oser, L., et al. 2010, ApJ, 725, 2312

Pastorello, N., et al. 2014, MNRAS, 442, 1003

Villumsen, J., 1983, MNRAS, 204, 219

Wyse, R. \& Silk, J. 1989, ApJ, 339, 700 